\title{
Network Coded Modulation for Two Way Relay Networks
}

\author{
Dr. Abdulkareem Abdulrahman Kadhim ${ }^{1}$, Karrar Abdulkhabeer Ali ${ }^{2}$ \\ ${ }^{1}$ College of Information Engineering / Al-Nahrain University \\ abdulkareem.a@coie-nahrain.edu.iq \\ ${ }^{2}$ College of Information Engineering / Al-Nahrain University \\ karrar315@yahoo.com
}

\begin{abstract}
Modern wireless networks use cooperative relaying to expand its coverage area. Wireless relaying allows mobile terminals to participate in transmission even when these terminals are not being the initial source or final destination. This can be achieved in different ways ranging from simple conventional relaying to more sophisticated coding at terminal and relay nodes to improve transmission performance. The proposed work here studied such system by simulating different coding techniques to be used in two way relay network coding (TWRC). Some of these techniques were previously proposed to improve transmission performance in conventional data communication systems. BER performance of different arrangements of coding and relaying schemes are evaluated using computer simulation tests. Ideal AWGN channel model and standard wireless channel models for wireless networks are considered in the simulation tests. The results have been shown that coding schemes such as Trellis Coded Modulation (TCM) and Repeat Accumulate (RA) codes are more suitable in two way relaying networks. Over AWGN, TCM-8PSK improves BER performance by at least $2 \mathrm{~dB}$ at high SNR for different relaying arrangements. For fading channels, this advantage is slightly reduced. Regenerative repeating at relay node seems to be a promising arrangement. It seems that there is no sense in using RA codes over fading channels due to its poor performance and relatively lower speed caused by repetitions.
\end{abstract}

Keywords: Physical Layer Network Coding, TWRC, Coding Gain, TCM, RA.

الخلاصة: تستخدم الشبكات اللاسلكية الحديثة طريقة اعادة الارسال لتوسيع مجال تغطيتها. إن معيدات الارسال اللاسلكي تسمح بتوسيع مجال تغطية الثبكة اللاسلكية.

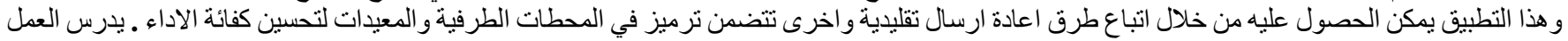

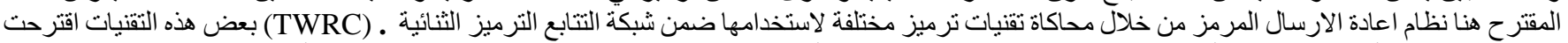

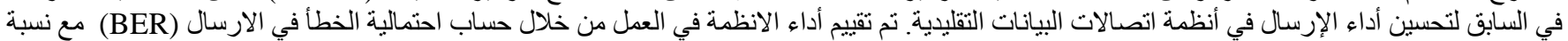

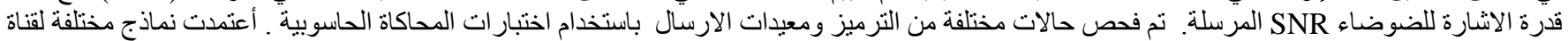

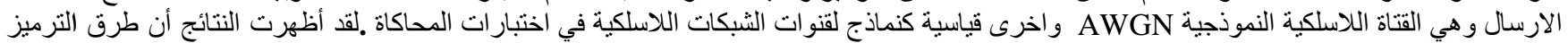

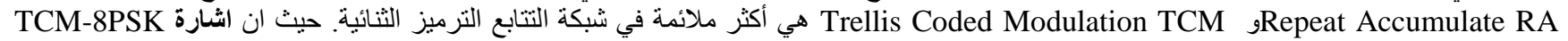

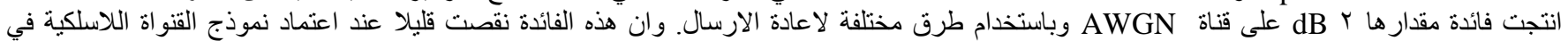

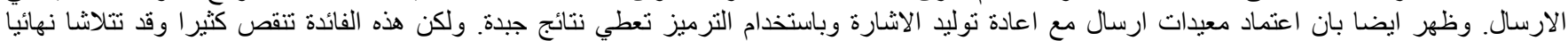

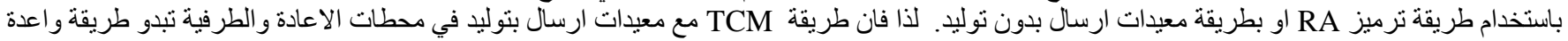

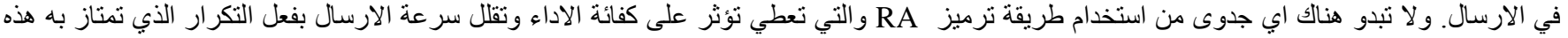
الطريق.

\section{INTRODUCTION}

\section{A. Two Way Relay Concepts}

Two way relay networks are three-nodes linear network in which two end nodes, Node-A and Node-B, want to communicate via a relay node Node-R as illustrated in Fig.1. There is no direct signal path between Node-A and Node-B. As an example is a satellite network in which Node-A and Node- $\mathrm{B}$ are the ground stations, and the relay Node- $\mathrm{R}$ is the satellite. The half-duplex constraint is often imposed on wireless communication systems to ease engineering design. With the half-duplex constraint, a node can't transmit and receive at the same time. With the half-duplex constraint, the relay in TWRC cannot receive from Node-A and Node-B and transmit to them at the same time slot.

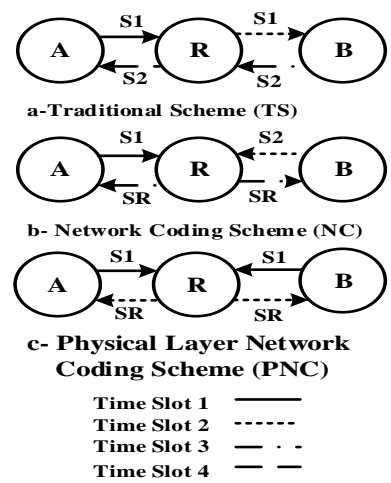

Figure 1:Transmission schemes for the two way relay channels

\section{B. Physical Layer Network Coding}

Physical layer network coding (PNC) is an approach used to improve transmission throughput of wireless networks in addition to some other advantages. PNC has been suggested 
to combat the limitations on networks devices and channels in classical networks [1]. With physical layer network coding, the relay will combine the symbols instead of only store-and-forward the output messages by routing, thus maximizing the overall system performance [2]. In its simplest form, PNC relies on intermediate nodes to combine (using X-OR) the incoming symbols from different source nodes and then to forward the linear encoded symbols to all destination nodes in a single transmission. Physical layer network coding can improve throughput, robustness complexity, reliability and security [3,4]. Each coding node serves as a relay node that combines the incoming symbols, from different source nodes, in one encoded packet to be transmitted to all destination nodes [5]. Fig-1 shows an example of simple network, where nodes A and B want to exchange their packets via a relay $(\mathrm{R})$. The traditional network in Fig-1(a) needs four transmissions to perform complete receptions of packets generated by source nodes $\mathrm{A}$ $\&$ B via the relay node $(\mathrm{R})$ to their intended destination nodes. On the other hand, the network coding (NC) defined by linear encoding of the incoming packets from source nodes, three transmissions are sufficient as in Fig-1(b). While with physical layer network coding that exploit the network coding operation that occurs naturally when electromagnetic waves are superimposed on one another need only two transmissions as in Fig-1(c). Thus, the best possible packet exchange throughput is two packets for every two slots, one in each direction. That is, $1 / 2$ packet per time slot per direction. Thus, when NC is compared with the traditional relay system as shown in Fig.1, it doubles the throughput of TWRC by reducing the needed time slots from four to three. It can be shown that $\mathrm{NC}$ has a throughput improvement of 33\% over traditional scheme (TS) [6]. While to compare PNC with the traditional scheme notice that PNC doubles the throughput of TWRC by reducing the needed time slots from four to two, and PNC can improve the performance $100 \%$ and $50 \%$ of TS and NC, respectively [6].

\section{RESEARCH BACKGROUND}

The present research is an attempt to combine physical layer network coding with modulation schemes so that possible increasing in coding gain can be achieved. Authors in [7] discussed physical-layer network coding in two-way decodeforward relay to obtain total minimum power consumption for the entire transmission process. Experimental results confirm that such scheme achieves much lower power consumption ratio than the existing schemes without PNC on the condition of the same system outage. In $[8,9]$ the twoways relaying system is considered, where two different users wish to exchange information with the help of a common relay node. To decode the superimposed signal from efficiently, they proposed a new scheme based on trellis coded modulation. The scheme improves the free distance of the coding sequence, thus more encoding gain is achieved.

A novel lattice coding scheme for two ways relay in [10] is introduced where every two nodes simultaneously communicate with each other through two relay nodes as well. Each node only communicates with its neighboring nodes then improving the achievable rates, and ensures that the messages traveling in each of the two directions fully utilize the relay's power. As a result the symmetric rate achieved by the proposed lattice coding scheme is within $1 / 2$ $\log 23 \mathrm{bit} / \mathrm{Hz} / \mathrm{s}$ of the symmetric rate capacity. Further developments were introduced by [11] to optimize the signal constellation for given coding scheme.

All above references were relied on using PNC in conjunction with TWRC to gain robustness and better performance. In the present work, a combined coding with modulation is to be used to show how the BER and coding gain of the network is improved.

The remaining parts of the paper are organized as follows: In the next section the model of the network used is to be described. The third section shows the simulation tests results in the form of error probability and the increase in coding gain versus channel SNR. The last section deals with the main concluding remarks of the work.

\section{SYSTEM MODEL}

\section{A. Coding Methods}

Two coding schemes are used here, the Repeat Accumulate (RA) code and Trellis Coded Modulation (TCM) schemes. The principles of both depend on adding redundancy to the transmitted data bits in order to reduce the errors at the receiver caused by the channel.

\section{1) Repeat Accumulate Coding}

The principle in the repeat accumulate is to take an input message and increase the redundancy to a certain level before convolutional encoding the message bits as depicted in Fig.2.

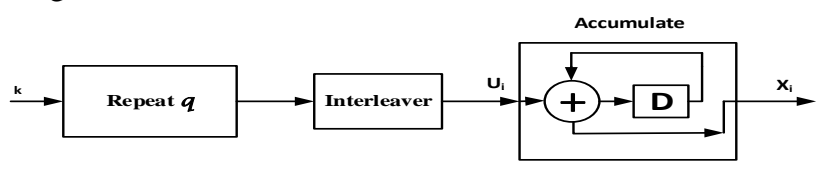

Figure 2: Repeat accumulate encoding scheme

Where $\mathrm{k}$ is the input bits and $\mathrm{q}$ is number of repetitions for each bit while D is memory. From Fig.2 one would observe that the message after repetition stage (each input bits are repeated $\mathrm{q}$ times) goes through the interleaver which interleaves the message, to introduce redundancy in the transmitted message. In this work $q$ is three, which choosing a representation three, is based on the fact that there is always a tradeoff between code rate and the throughput of a communication system. The following stage is a convolutional encode called the accumulator whose output is the coded sequence. The encoder in this scheme has memory and so the output of the scheme will depend on both the previous and current input value (message bits), and the encoder or the accumulator exhibits a recursive nature so the output follows the pattern below; 
$\mathrm{x} 1=\mathrm{u} 1$

$\mathrm{x} 2=\mathrm{u} 1+\mathrm{u} 2$

$\mathrm{x} 3=\mathrm{u} 1+\mathrm{u} 2+\mathrm{u} 3$

$$
\mathrm{x}_{\mathrm{n}}=\mathrm{u}_{1}+\mathrm{u}_{2}+\mathrm{u}_{3}+\mathrm{u}_{4}+\ldots . .+\mathrm{u}_{\mathrm{n}}=\sum_{1}^{n}\left(u_{n} \bmod 2\right)
$$

where ui is the ith input (i.e output of the interleaver at instant $\mathrm{i}$ ) and $\mathrm{xj}$ is the output coded bit of the encoder at time j.

The decoder follows the process of encoding. Thus the output, in the absence of channel effects, follows the pattern below;

$$
\begin{aligned}
& \bar{u}_{1}=x_{1} \\
& \bar{u}_{2}=x_{2}+x_{1} \\
& \bar{u}_{3}=x_{3}+x_{2} \\
& \bar{u}_{n}=x_{n}+x_{n 1}
\end{aligned}
$$

where $\mathrm{x}$ is the input symbol to the convolutional decoder after channel and $\mathrm{u}^{-}$is output symbol from decoder.

Then, the next step is the deinterleaver which is performing an inverse permutation to that of the interleaver [11]. After that the choice for bit is based on a majority logic decision on the group of three deinterleaved bits at the output of the deinterleaver [11]

\section{2) Trellis Coded Modulation}

TCM combines the choice of a modulation scheme with that of a convolutional code together to improve the reliability of a digital transmission system and for the purpose of gaining noise immunity over uncoded transmission without expanding the signal bandwidth or increasing the transmitted power or reduction of data rate. At TCM the mapper is used for mapping the source symbols into signals, this known as set partition [12] which provide redundancy for coding, and to design coding and signal mapping functions jointly to maximize the "free distance" (minimum Euclidean distance) between coded signal sequences. This allowed the construction of modulation codes whose free distance is significantly exceeding the minimum distance of the uncoded modulation signals, at the same information rate, bandwidth, and signal power.

In this work, TCM simulated encoder consists of the convolutional trellis encoder (rate $=2 / 3$ ) and a memoryless mapper [13,14]. At the receiver the decoder is Viterbi type decoder that works on the received samples to produce the originally transmitted data bits, as shown in Fig.3.

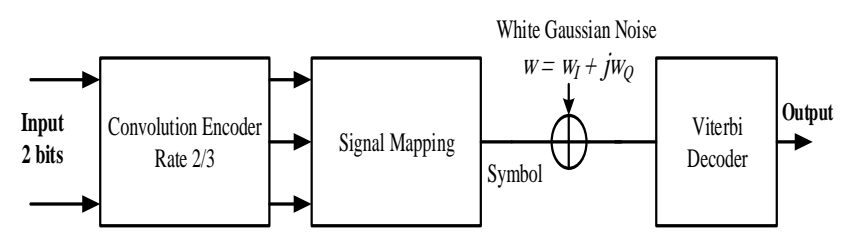

Figure 3: Trellis coded modulation model for coded 8PSK signal
The structure of the convolutional encoder decides the relevant construction of the trellis diagram [15], which is very important in the Viterbi decoding.

The encoding process, at each time instance $t$, the TCM encoder, as depicted in the Fig.3, receives two information bits and generates three coded bits which define one symbol. The chosen symbol is sent through the channel (AWGN, Flat fading and SUI-3) at times $t$ and $t+1$. The symbols are represented as the complex numbers. The number of the information bits at the input of the encoder determines which constellation and which code would be used.

While the decoding process, at the receiver a noisy complex number is received at each instance time t. The Viterbi algorithm should be used to decode them [16].

\section{B. Transmission Schemes}

In the TWRC, we have two nodes nodel and node 2 exchange its information's with the help of Relay node. We assume all nodes are half-duplex, (i.e., each node cannot receive and transmit simultaneously). This is an assumption arising from practical considerations because it is difficult for wireless nodes to remove the strong interference (channel effect) of its own transmitting signal from the received signal. We also assume that there is no direct link between node 1 and node 2.

Throughout this paper, all systems used implement in three different transmission scenarios, with no physical layer network coding being used (BPSK or QPSK), with physical layer network coding (point-to-point) and physical layer network coding with two-way relay transmission. (Regenerative and Non-regenerative Repeaters are used at relay node).

1) Point to Point

At this type of transmission (point-to-point), symbols coded or uncoded, each node send its coded symbols to another node simultaneously. Then decoding operation occurs at each node. Of course, each node already knew its data. So that, each node compares its self-information with the decoded composed symbols.

\section{2) Non-Regenerative Repeater}

When Non-regenerative Repeater technique is used, each node (source) sends its coded information's then the relay node receive noised information, noised because channel, for both nodes where it role is only made sum operation for received information which does not made any coding or decoding operation for received information, after sum operation relay will broadcast its information's to end nodes, at this point also here noise (channel) be added to data prior to receiving by both nodes. When two nodes receive data from relay, each node perform equalization and decoding operation according to type a system used, regard of that all nodes uses same encoder and decoder.

\section{3) Regenerative Repeater}

When the Regenerative Repeater technique is used, each node send its coded information's to relay, here relay perform decoding operation of received data for each node, then perform sum operations for decoded data for both nodes. After that it also made coding operation for summed result of two nodes. Then broadcast coded data to both 
nodes, at this point each node perform decoding operation for received data from relay, then each node compare its selfinformation with the decoded composed symbols which each node already knew its data.

\section{Transmission Chanel Models}

\section{1) AWGN Channel}

The AWGN channel function is to add white Gaussian noise to the input signals. The term noise is the unwanted electrical signals that are always present in electrical systems and the additive means the noise is superimposed to the signal that tends to mask the signal where it will limit the receiver ability to make correct symbol decisions [17].

\section{2) Flat Fading Channel}

The delays associated with different signal paths in a multipath fading channel change in an unpredictable manner and can only be characterized statistically. A fading channel generally reduces the ratio of the signal power to the noise power at the receiver, thus also reducing the transmission capacity. Fading can be represented as a convolution of the transmitted signal with the channel impulse response (h), as illustrated in Fig.4.

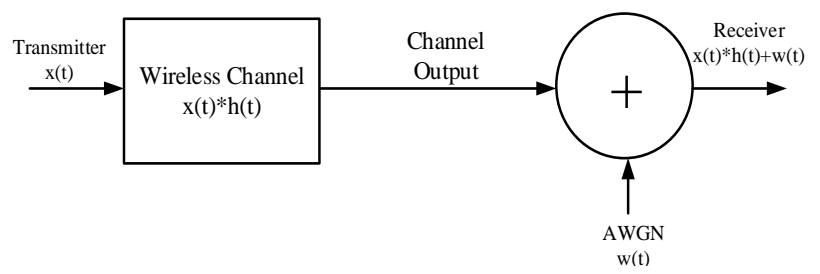

Figure 4 System model for transmission through a channel with additive white Gaussian noise.

Flat fading can be obtained from zero-mean complex Gaussian processes. Simply adding the Gaussian random variables and taking the square root (envelope) gives a Rayleigh distributed process. The channel also adds AWGN noise to the signal samples after it suffers from Rayleigh Fading as shown in Fig.3-7. The received signal y can be represented as [18].

$\mathrm{y}=$

$\mathrm{h} * \mathrm{x}$

W

(3)

where $\mathrm{w}$ is the noise contributed by AWGN which is Gaussian distributed with zero mean and fixed variance and $\mathrm{h}$ (fading amplitude) is the Rayleigh Fading response with zero mean and unit variance.

\section{3) SUI-3 channel}

Stanford University Interim (SUI-3) channel model is used in the work. SUI-3 channel model represents broadband fixed wireless channel normally considered for IEEE802.16.a and with three paths channel. The maximum Doppler shift for all paths is $0.5 \mathrm{~Hz}$. Path delays are $0,0.4$, and $0.9 \mu$ s for path one, two, and three respectively. Gains of paths are $0,-5$, and $-10 \mathrm{~dB}$ for path one, two, and three respectively, and one antenna for each of transmitter and receiver [19]. Details of the actual channel modeling and complete system simulation can be found elsewhere [16].

\section{Simulation Results \& AsSessment}

The performance of the proposed system has been measured in terms of Bit Error Rate (BER) versus the signal-to-noise power ratio (SNR). The signal-to-noise power ratio is given by Eb / N0 (in dB). Eb is the transmitted signal energy per bit and N0 is single sided power spectral density of the AWGN.

In addition to the original TCM-8PSK and RA-BPSK signals, the cases of uncoded BPSK and QPSK-uncoded signals are also considered in transmission for the sake of comparison. These signals are tested with two different repeater schemes; Regenerative and Non-regenerative repeaters. Fig.5 show the BER performances of different systems considered in the work. In parts $\mathrm{a}, \mathrm{b}$ and $\mathrm{c}$ AWGN, Flat fading and SUI-3 channels are considered, respectively. In the case of AWGN channel the comparison on the bases of transmission schemes (repeater type) shows that the regenerative repeater based uncoded (BPSK and QPSK) modulation gains about $9 \mathrm{~dB}$ for BER of about 10-5 relative to the corresponding signals based on non-regenerative scheme. For flat fading channel, this advantage is about 10 $\mathrm{dB}$ (at BER of 10-3) and about $5 \mathrm{~dB}$ in the case of SUI-3 channel. This behavior is clear also with the coding scheme RA-BPSK . The corresponding gain is about 7, 1, and $3 \mathrm{~dB}$ for AWGN, flat fading, and SUI3 channel models, respectively. Further, these gains are changed to about 6,1 , and $5 \mathrm{~dB}$ for the case of TCM-8PSK, respectively.

As an overall comparison for all systems operating over the three channels; the system with single link (point to point) has the best performance at high SNR as compared to the corresponding systems with two-way relay nodes or relaying scheme, whether it is a regenerative type or not. The use of regenerative scheme show better performance over nonregenerative one. When TCM is used with AWGN channel, the BER performance is improved. For coding schemes (RA or TCM) with Flat fading channel, the BER performance is improved. This is also clear when comparing QPSK with TCM-8PSK for the same transmission scenario and it is also clear when comparing BPSK to RA-BPSK. When TCM is used over SUI-3 channel, the BER performance is improved as it is clear when comparing QPSK with TCM-8PSK for the same transmission scenario. All tested systems here are operating at the same bandwidth but may have different bit rates [16]. TCM-8PSK and QPSK have the same bit rate, while the bit rate of uncoded BPSK is three times the bit rate of RA-BPSK.

Over SUI-3 channel, it is noticed that the advantage of TCM8PSK system is not reserved when compared to the corresponding advantage over AWGN channel or flat fading channel for all schemes tested. This is due to accumulated channel distortion caused by the relaying operation which greatly affecting the distance properties of TCM systems [20]. 


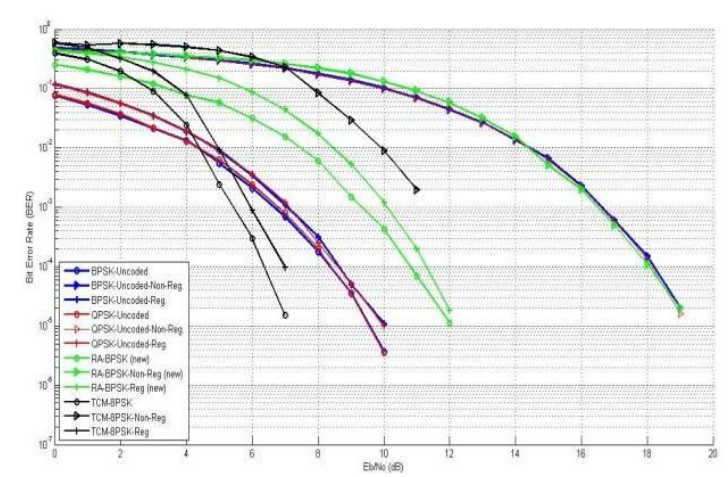

a. Different systems over AWGN channel

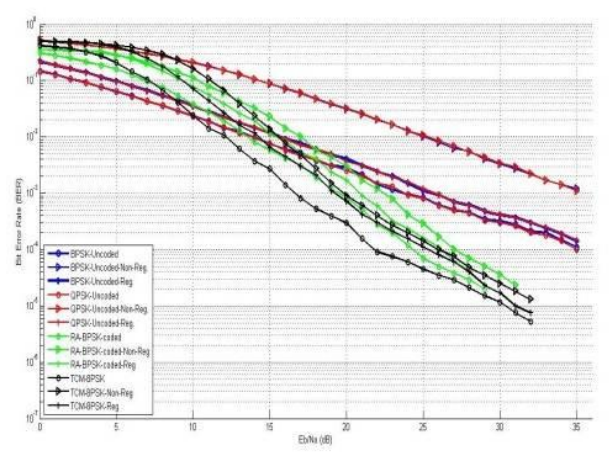

b. Different systems over Flat fading channel

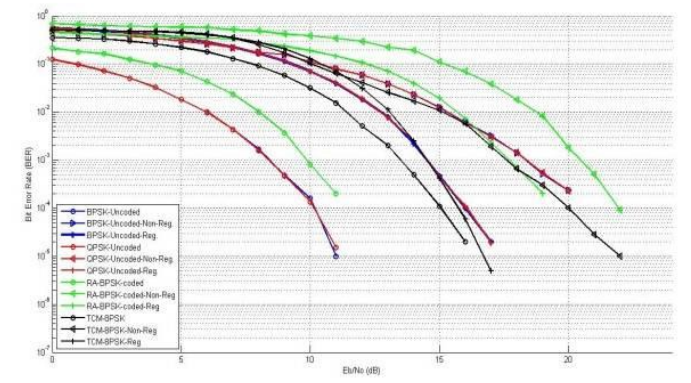

c. Different systems over SUI-3 channel

Figure 5: Bit error rate performances

\section{Conclusions}

In this paper, physical-layer network coding (PNC) was investigated for two-way relay network using both (RA) code and (TCM) PSK signaling. The advantage of employing modulation and channel coding with network coding in the form of PNC shows encouraging results. On the basis of present investigation and simulation results one can conclude that combining modulation with PNC is a promising option for improving the performance of communication system. Using regenerative repeater with coding at the relay node is an effective method to improve transmission. TCM-8PSK signal provided best performance over all the systems, tested in this work, in terms of both BER and transmission speed. RA-BPSK offered simple coding and decoding processes when compared to uncoded counterpart (BPSK), while its transmission speed for given bandwidth is much less than those of the uncoded BPSK modulation or TCM-8PSK signal. Further degradation in performance of RA occurred over fading channels.

\section{REFERENCES}

[1]G. Kramer, "Lecture on Network Coding and Information Theory,” Alcatel Lucent, Sep. 2007.

[2]R. Ahlswede, N. Cai, S.-Y.R.Li, and R.W.-H. Yrung, "Network Information Flow," IEEE Trans. Inf. Theory, Vol. 46, No.4, pp. 1204-1216, July 2000.

[3]S. Katti, "Network Coded Wireless Architecture," Ph.D. Thesis, Department of Electrical Engineering and Computer Science, Massachusetts Institute of Technology, Aug. 2008.

[4]C. Fragouli \& E. Soljanin, "Network Coding Fundamentals", Foundations and Trends in Networking, Boston, Vol. 2, Issue 1, 2007.

[5]T. Ho \& D. Lun, "Network Coding: An Introduction," Cambridge University Press, New York, 2008.

[6]S. Zhang, S. Liew, P. Lam, "Hot Topic: Physicallayer Network Coding," Proceedings of the 12th annual international conference on Mobile computing and networking, '06, pp. 358-365, New York, USA, Sept. 2006.

[7]M. Zhao, Y. Zhou, D. Ren and Y. Yang, "A minimum power consumption scheme for two-way relay with physical-layer network coding," IEEE $2^{\text {nd }}$ International Conference on Network Infrastructure and Digital Content, pp. 704-708, Beijing, China, Sept.2010.

[8]Chen, Z. Zheng, B.Ji, X.Zhang and W.liang, "Joint design of physical layer network coding and channel decoding based on trellis coded modulation in two-way relay channel," IEEE International Conference on Wireless Commun. and Signal Processing, pp. 1-5, ISBN: 978-1-4577-1009-4, Nanjing, China, Nov. 2011.

[9] T. Koike-Akino, P. Popovski \& V. Tarokh, "Optimized Constellations for Two-Way Wireless Relaying with Physical Network Coding", Proceedings of the Global Communications 
Conference, 2008. GLOBECOM 2008, New

Orleans, LA, USA, 30 November - 4 December

2008.

[10] Y. Song, N. Devroye, H. R. Shao and C. Ngo, "Lattice Coding for the Two-way Two-relay Channel", submitted to IEEE Transactions on Information Theory, Cornell University, USA, Dec. 2012.

[11] B.Motsie, "Matlab toolbox for simulation of repeataccumulate codes", M.Sc. Thesis, Newcastle University, Australia, 2005.

[12] W. Chen, L. Hanzo, \& Z. Cao, "Network Coded Modulation for Two-Way Relaying", Proceedings of IEEE Conference WCNC, 2011

[13]E. Krouk and S. Semenov, "Modulation and Coding Techniques in Wireless Communications," John Wiley \& Sons Ltd., United Kingdom, 2011.

[14] J-G. Proakis and M. Salehi, "Communication Systems Engineering," Prentice-Hall,Inc, $2^{\text {nd }}$ edition, ISBN 0-13-061793-8, USA, August 2002.

[15] G. Ungerboeck, "Trellis coded Modulation with Redundant Signal Sets PartII: State of the Art", IEEE, communications magazine, Vol. 25, No.2, pp. 12-21, Feb.1987

[16]K. A. Ali, "Network Coded Modulation for TwoWay Relay Networks", M.Sc. Thesis, Al-Nahrain University, Iraq, Sept. 2013.

[17] Bernard Sklar, "Digital Communications: Fundamentals and Applications", Prentice- Hall, 2nd Edition, 1991.

[18] M. Franceschini, G. Ferrari and R. Raheli, "LDPC Coded Modulations," Springer-Verlag Berlin Heidelberg, Library of Congress Control Number: 2009921992, ISBN 978-3-540-69455-7, 2009.

[19] V. Erceg, K.V. S. Hari, M.S. Smith, D.S. Baum, K.P. Sheikh, C.Tappenden, J.M. Costa, C. Bushue, A. Sarajedini, R. Schwartz, and D.Branlund, Channel Models for Fixed Wireless Applications", IEEE802.16 Broadband Wireless Access Working Group, July, 2001.

[20]A. P. Clark \& A-K.A-R.Kadhim, "Detection of Coded and Distorted QAM Signals", Journal of IERE, Vol. 58, No. 4, Aug. 1988. (U.K). 